\title{
Conformal deformations to scalar-flat metrics with constant mean curvature on the boundary
}

\author{
Fernando C. Marques
}

\begin{abstract}
Let $\left(M^{n}, g\right)$ be a compact manifold with boundary, with finite Sobolev quotient $Q\left(M^{n}, \partial M\right)$. We prove that there exists a conformal deformation which is scalar-flat and has constant boundary mean curvature, if $n=4$ or 5 and the boundary is not umbilic. In particular, we prove such existence for any smooth and bounded open set of the Euclidean space, finishing the remaining cases of a theorem of J.F. Escobar.
\end{abstract}

\section{Introduction}

The classical Riemann mapping theorem is one of the most famous results in Mathematics. It states that any simply connected, proper domain of the plane is conformally diffeomorphic to a disk. This theorem is certainly false in higher dimensions, since the only bounded open subsets of $\mathbb{R}^{n}, n \geq 3$, that are conformally diffeomorphic to Euclidean balls are the Euclidean balls themselves. Nevertheless, we could ask how close to a ball one can get by means of a conformal deformation of the metric. In that spirit, Escobar proved in [7] (the proof in dimension 6 appeared in [11]) the following beautiful result:

Theorem 1.1. (Escobar) Let $\Omega \subset \mathbb{R}^{n}$ be a smooth bounded domain of the Euclidean space, $n \neq 4,5$. Then there exists a smooth function $u>0$ such that $\tilde{g}_{i j}=u^{4 /(n-2)} \delta_{i j}$ is scalar-flat and has constant mean curvature on the boundary $\partial \Omega$.

In fact, this is a special case of the following problem:

Yamabe problem. Let $\left(M^{n}, g\right)$ be an n-dimensional, compact, Riemannian manifold with boundary $\partial M, n \geq 3$. Is there a conformally related metric $\tilde{g}$ with zero scalar curvature and boundary $\partial M$ of constant mean curvature?

That general question was addressed for the first time by Escobar in [7], where he gave an affirmative answer for a large class of manifolds (see 
Theorem 2 in that paper). The present author studied a higher dimensional case in [19], where the Weyl tensor on the boundary plays a natural role. We should also note that the classical Yamabe problem, for manifolds without boundary, was completely solved after the works of Yamabe [22], Trudinger [21], Aubin [2] and Schoen [20].

The problem we are interested in is equivalent, in analytical terms, to finding a smooth positive solution to the nonlinear boundary-value problem:

$$
\begin{aligned}
& \Delta_{g} u-\frac{n-2}{4(n-1)} R_{g} u=0 \text { in } M, \\
& \frac{\partial u}{\partial \eta}+\frac{n-2}{2} h_{g} u=\frac{n-2}{2} c u^{n / n-2} \text { on } \partial M
\end{aligned}
$$

where $\Delta_{g}$ denotes the Laplace-Beltrami operator of the metric $g, R_{g}$ is the scalar curvature, $h_{g}$ is the mean curvature of the boundary with respect to $g$, $\eta$ is the outward unit normal vector to $\partial M$ and $c$ is a constant. In fact, given a solution $u$ to problem (1.1), the metric $\tilde{g}=u^{4 /(n-2)} g$ is scalar-flat and its boundary mean curvature is equal to $c$. Note that the Equations (1.1) are specially interesting due to the boundary nonlinearity.

The solutions to Equations (1.1) can also be seen as critical points of the functional

$$
Q(\phi)=\frac{\int_{M}\left(|\nabla \phi|^{2}+(n-2) / 4(n-1) R_{g} \phi^{2}\right) d v_{g}+(n-2) / 2 \int_{\partial M} h_{g} \phi^{2} d \sigma_{g}}{\left(\int_{\partial M}|\phi|^{2(n-1) /(n-2)} d \sigma_{g}\right)^{(n-2)} /(n-1)} .
$$

Here, $d v_{g}$ and $d \sigma_{g}$ denote the volume forms of $M$ and $\partial M$, respectively.

In [7], Escobar introduced the Sobolev quotient

$$
Q(M, \partial M)=\inf \left\{Q(\phi): \phi \in C^{1}(\bar{M}), \phi \not \equiv 0 \text { on } \partial M\right\}
$$

proving that it is conformally invariant and always satisfies

$$
Q(M, \partial M) \leq Q\left(B^{n}, \partial B^{n}\right)
$$

where $B^{n}$ denotes the unit ball in $\mathbb{R}^{n}$ endowed with the Euclidean metric.

Under the hypothesis that $Q(M, \partial M)$ is finite (which is the case if $\left.R_{g} \geq 0\right)$, he also showed that the strict inequality

$$
Q(M, \partial M)<Q\left(B^{n}, \partial B^{n}\right)
$$

assures the existence of a minimizing solution to problem (1.1). 
The main result of the present paper is as follows.

Theorem 1.2. Let $\left(M^{n}, g\right)$ be a compact manifold with nonempty boundary, $n=4$ or 5 . Assume the boundary $\partial M$ is not umbilic. Then

$$
Q\left(M^{n}, \partial M\right)<Q\left(B^{n}, \partial B\right)
$$

If $Q\left(M^{n}, \partial M\right)$ is finite, then there exists a smooth function $u>0$ such that $\tilde{g}=u^{4 /(n-2)} g$ is scalar-flat and has constant boundary mean curvature.

We should point out that $Q\left(M^{n}, \partial M\right)$ could be $-\infty$, as it is the case of manifolds obtained by deleting a small geodesic ball from a closed manifold of negative scalar curvature (see [9] for details).

In the case of a smooth bounded domain $\Omega \subset \mathbb{R}^{n}$, we know that the boundary is not umbilic unless the domain is a ball (in which case the existence is trivial). We also have that $Q(\Omega, \partial \Omega)$ is finite because the Euclidean metric is scalar-flat.

Therefore, we finish the remaining cases of Theorem 1.1 as the following corollary:

Corollary 1.3. Theorem 1.1 is also true in dimensions 4 and 5 .

The proof of Theorem 1.2 is explicitly based on constructing a function $\phi$, with support in a small halfball around a nonumbilic point $P \in \partial M$, such that

$$
Q(\phi)<Q\left(B^{n}, \partial B^{n}\right)
$$

The usual strategy in this kind of problem (which goes back to Aubin, [2]) consists in defining the function $\phi$, in the small halfball, as one of the standard entire solutions to the corresponding Euclidean equations. In our context those are

$$
U_{\varepsilon}(x, t)=\left(\frac{\varepsilon}{(\varepsilon+t)^{2}+|x|^{2}}\right)^{(n-2) / 2} .
$$

The next step would be to expand the quotient of $\phi$ in powers of $\varepsilon$ and, by exploiting the local geometry around $P$, show that the inequality (1.2) holds if $\varepsilon$ is small. In order to simplify the asymptotic analysis, we use conformal Fermi coordinates (introduced in [19]), which play the same role the conformal normal coordinates (see [18]) did in the classical Yamabe problem. 
The difficulty arises because, when $3 \leq n \leq 5$, the first correction term in the expansion has the wrong sign. When $n=3$, Escobar proved the strict inequality by applying the Positive Mass Theorem (a global construction originally due to Schoen, [20]). This argument does not work when $4 \leq n \leq$ 5 because the metric is not sufficiently flat around the nonumbilic point $P \in \partial M$.

Our point of view in this article is that the test functions $U_{\varepsilon}$ are not optimal in dimensions 4 and 5 , but the problem should still be local. This kind of phenomenon does not appear in the classical solution of the Yamabe problem for manifolds without boundary, although we should say that perturbed test functions have already been used in the work of Hebey and Vaugon $([17])$.

In order to prove the strict inequality (1.2), we introduce

$$
\psi_{\varepsilon}(x, t)=\varepsilon^{(n-2) / 2} h_{i j} x^{i} x^{j} t\left(\frac{1}{(\varepsilon+t)^{2}+|x|^{2}}\right)^{n / 2},
$$

where $h_{i j}$ are the coefficients of the second fundamental form at $P$. Our test function $\phi$ is defined as

$$
\phi=U_{\varepsilon}+\psi_{\varepsilon}
$$

around $P \in \partial M$.

When $n=5$, this solves the problem because the first correction term in the expansion of $Q(\phi)$ is strictly negative, but if $n=4$ one can check that the term actually vanishes. In order to deal with that case, we need to work with a small perturbation $\phi=U_{\varepsilon}+\psi_{\varepsilon}+\delta T$.

The motivation for the definition (1.3) came from blowup analysis ideas, by determining good enough approximations of solutions concentrating at a boundary point. We think it should be possible to apply this sort of reasoning to other similar problems.

Other works concerning conformal deformation on manifolds with boundary include $[1,4,5,8,10,12-16]$.

\section{Preliminaries and statements}

Let $\left(M^{n}, g\right)$ be an $n$-dimensional compact Riemannian manifold with boundary, $n \geq 3$. In what follows, let $R_{g}$ denote the scalar curvature of the metric $g$, and let $h_{g}$ denote the mean curvature of $\partial M$.

We are interested in finding a conformal deformation $\tilde{g}=u^{4 /(n-2)} g$, which is scalar-flat and has constant mean curvature on the boundary. This 
geometric problem is equivalent to finding a constant $c \in \mathbb{R}$ and a positive solution $u$ to

$$
\begin{array}{llr}
\Delta_{g} u-\frac{n-2}{4(n-1)} R_{g} u=0 & \text { in } & M, \\
\frac{\partial u}{\partial \eta}+\frac{n-2}{2} h_{g} u=\frac{n-2}{2} c u^{n /(n-2)} & \text { on } & \partial M .
\end{array}
$$

The operator $L_{g}=\Delta_{g}-\frac{n-2}{4(n-1)} R_{g}$ is called the conformal Laplacian of $g$.

In order to obtain the variational formulation of problem (2.1) we need to introduce the Sobolev quotient of $M$.

Given $\phi \in H^{1}(M)$, define

$$
E(\phi)=\int_{M}\left(\left|\nabla_{g} \phi\right|^{2}+\frac{n-2}{4(n-1)} R_{g} \phi^{2}\right) d v_{g}+\frac{n-2}{2} \int_{\partial M} h_{g} \phi^{2} d \sigma,
$$

and also

$$
Q(\phi)=\frac{E(\phi)}{\left(\int_{\partial M}|\phi|^{2(n-1) /(n-2)} d \sigma\right)^{(n-2) /(n-1)}} .
$$

One can now check that solutions to the Equations (2.1) are the positive functions which are critical points of $Q$.

The Sobolev quotient $Q(M, \partial M)$ is defined as

$$
Q(M, \partial M)=\inf \left\{Q(\phi): \phi \in C^{1}(\bar{M}), \phi \not \equiv 0 \text { on } \partial M\right\} .
$$

It is not difficult to see [7] that the Sobolev quotient is a conformal invariant.

It follows from the work in [7] that whenever

$$
-\infty<Q\left(M^{n}, \partial M\right)<Q\left(B^{n}, \partial B\right)
$$

there exists a positive minimizer of $Q$. This minimizer is automatically a solution to the problem (2.1).

The number $Q\left(B^{n}, \partial B\right)$ also appears as the best constant in the Sobolevtrace inequality:

$$
\begin{aligned}
& \left(\int_{\partial \mathbb{R}_{+}^{n}}|\phi|^{2(n-1) /(n-2)} d x\right)^{(n-2) /(n-1)} \leq \frac{1}{Q\left(B^{n}, \partial B\right)} \int_{\mathbb{R}_{+}^{n}}|\nabla \phi|^{2} d x d t, \\
& \text { for every } \phi \in H^{1}\left(\mathbb{R}_{+}^{n}\right) .
\end{aligned}
$$

Here $\mathbb{R}_{+}^{n}=\left\{(x, t) \in \mathbb{R}^{n}: t \geq 0\right\}$. 
It was proven by Escobar [6], and independently by Beckner [3], that the equality is achieved by

$$
U_{\varepsilon}(x, t)=\left(\frac{\varepsilon}{(\varepsilon+t)^{2}+|x|^{2}}\right)^{(n-2) / 2},
$$

which is a solution to the boundary-value problem:

$$
\begin{array}{lr}
\Delta U_{\varepsilon}=0 & \text { in } \mathbb{R}_{+}^{n}, \\
\frac{\partial}{\partial t} U_{\varepsilon}=-(n-2) U_{\varepsilon}^{n /(n-2)} & \text { on } \partial \mathbb{R}_{+}^{n} .
\end{array}
$$

One can check, using integration by parts, that

$$
\int_{\mathbb{R}_{+}^{n}}\left|\nabla U_{\varepsilon}\right|^{2} d x d t=(n-2) \int_{\partial \mathbb{R}_{+}^{n}} U_{\varepsilon}^{2(n-1) / n-2} d x,
$$

and also

$$
Q\left(B^{n}, \partial B^{n}\right)=(n-2)\left(\int_{\partial \mathbb{R}_{+}^{n}} U_{\varepsilon}^{2(n-1) / n-2} d x\right)^{1 / n-1}
$$

\section{Estimating the Sobolev quotient}

In this section, we will prove Theorem 1.2 by constructing a function $\phi$ with

$$
Q(\phi)<Q\left(B^{n}, \partial B\right)
$$

Let $P \in \partial M$ be a nonumbilic point, and choose geodesic normal coordinates $\left(x_{1}, \ldots, x_{n-1}\right)$ on the boundary, centered at $P$. We say that $\left(x_{1}, \ldots, x_{n-1}, t\right)$ are the Fermi coordinates of the point $\exp _{x}(t \eta(x)) \in M$, where $\eta(x)$ denotes the inward unit vector normal to the boundary at $x \in \partial M$ and $t \geq 0$ is small. It is easy to see that in these coordinates $g_{t t} \equiv 1$ and $g_{t i} \equiv 0$. The expansion of the coefficients $g_{i j}$ up to second order is given by (see [19, Lemma 2.2]):

$$
\begin{aligned}
& g_{i j}=\delta_{i j}-2 h_{i j} t \\
& -\frac{1}{3} \bar{R}_{i k j l} x_{k} x_{l}-2 h_{i j, k} t x_{k}+\left(-R_{t i t j}+h_{i s} h_{s j}\right) t^{2}+O\left(|(x, t)|^{3}\right) .
\end{aligned}
$$

All the coefficients are computed at $P$, where $h_{i j}$ denotes the second fundamental form with respect to the inward unit normal, and $R_{t i t j}, \bar{R}_{i k j l}$ 
denote components of the full Riemannian curvature tensors of $M$ and $\partial M$, respectively.

Since the Sobolev quotient $Q\left(M^{n}, \partial M\right)$ is a conformal invariant, we can assume (see [19, Section 3]) there exist conformal Fermi coordinates, i.e., Fermi coordinates $\left(x_{1}, \ldots, x_{n-1}, t\right)$ centered at $P$ such that

$$
d v_{g}=\left(1+O\left(|(x, t)|^{N}\right)\right) d x d t,
$$

where $N$ is arbitrarily large.

In general, from the expansion (3.1),

$\operatorname{det} g=1-2 H t$

$$
-\frac{1}{3} \bar{R}_{k l} x_{k} x_{l}-2 H_{, k} t x_{k}+\left(-R_{t t}-|h|^{2}+2 H^{2}\right) t^{2}+O\left(r^{3}\right) .
$$

Here $H$ denotes the mean curvature, $|h|^{2}=\sum_{i, j}\left(h_{i j}\right)^{2}$, and $R_{t t}, \bar{R}_{k l}$ denote components of the Ricci tensors of $M$ and $\partial M$, respectively.

Hence, in conformal Fermi coordinates, we have $H=H_{, k}=\bar{R}_{k l}=0$, and $R_{t t}=-\sum_{i, j}\left(h_{i j}\right)^{2}$ at $P$.

In what follows $B_{\rho}^{+}=\left\{(x, t) \in \mathbb{R}_{+}^{n}:|x|^{2}+t^{2} \leq \rho^{2}\right\}, S_{\rho}^{+}=\left\{(x, t) \in \mathbb{R}_{+}^{n}:\right.$ $\left.|x|^{2}+t^{2}=\rho^{2}\right\}$ and $B_{\rho}^{n-1}=\left\{(x, 0) \in \mathbb{R}^{n}:|x|^{2} \leq \rho^{2}\right\}$.

Fix $A \in \mathbb{R}, \rho_{0}>0$ and $\varepsilon>0$.

If

$$
\psi_{\varepsilon}(x, t)=A \varepsilon^{(n-2) / 2} h_{j k} x_{j} x_{k} t\left(\frac{1}{(\varepsilon+t)^{2}+|x|^{2}}\right)^{n / 2},
$$

define

$$
u_{\varepsilon}=U_{\varepsilon}+\psi_{\varepsilon} \quad \text { for }(x, t) \in \mathbb{R}_{+}^{n},
$$

where $U_{\varepsilon}$ is as in the previous section.

Note that

$$
u_{\varepsilon}(x, t)=(1+O(r)) U_{\varepsilon}(x, t) .
$$

Hence, if $\rho_{0}$ is sufficiently small and $A$ is fixed

$$
\frac{1}{2} U_{\varepsilon} \leq u_{\varepsilon} \leq 2 U_{\varepsilon} \quad \text { in } B_{2 \rho_{0}}^{+} .
$$

Let $\eta(\rho)$ be a smooth cut-off function satisfying $\eta(\rho)=1$ for $\rho \leq \rho_{0}$, $\eta(\rho)=0$ for $\rho \geq 2 \rho_{0}, 0 \leq \eta \leq 1$ and $\left|\eta^{\prime}(\rho)\right| \leq C \rho_{0}^{-1}$ if $\rho_{0} \leq \rho \leq 2 \rho_{0}$.

Define

$$
\phi=\eta u_{\varepsilon} .
$$

Let us first estimate $E_{g}(\phi)$ on $A_{\rho_{0}}^{+}=B_{2 \rho_{0}}^{+} \backslash B_{\rho_{0}}^{+}$. 
In what follows, we will denote by $C$ different positive constants.

First observe

$$
|\nabla \phi|_{g}^{2} \leq C|\nabla \phi|^{2} \leq C\left(|\nabla \eta|^{2} u_{\varepsilon}^{2}+\eta^{2}\left|\nabla u_{\varepsilon}\right|^{2}\right)
$$

Now

$$
\begin{aligned}
\int_{A_{\rho_{0}}^{+}}|\nabla \eta|^{2} u_{\varepsilon}^{2} d x d t & \leq C \rho_{0}^{-2} \int_{A_{\rho_{0}}^{+}} U_{\varepsilon}^{2} d x d t \\
& \leq C \rho_{0}^{-2} \varepsilon^{n-2} \int_{A_{\rho_{0}}^{+}}\left(\frac{1}{t^{2}+|x|^{2}}\right)^{n-2} d x d t \\
& =C \rho_{0}^{-2} \varepsilon^{n-2} \int_{\rho_{0}}^{2 \rho_{0}} \rho^{3-n} d \rho \\
& \leq C \varepsilon^{n-2} \rho_{0}^{2-n}
\end{aligned}
$$

We also have

$$
\int_{A_{\rho_{0}}^{+}} \eta^{2}\left|\nabla u_{\varepsilon}\right|^{2} d x d t \leq C \varepsilon^{n-2} \int_{\rho_{0}}^{2 \rho_{0}} \rho^{1-n} d \rho \leq C \varepsilon^{n-2} \rho_{0}^{2-n} .
$$

The other terms are estimated as follows:

$$
\int_{A_{\rho_{0}}^{+}} R_{g} \phi^{2} d x d t \leq C \int_{A_{\rho_{0}}^{+}} U_{\varepsilon}^{2} d x d t \leq C \varepsilon^{n-2} \rho_{0}^{4-n}
$$

and

$$
\int_{A_{\rho_{0}}^{+} \cap \partial M} h_{g} \phi^{2} d x \leq C \int_{A_{\rho_{0}}^{+} \cap \partial M} U_{\varepsilon}^{2} d x \leq C \varepsilon^{n-2} \rho_{0}^{3-n} .
$$

We conclude that

$$
E_{M \backslash B_{\rho_{0}}^{+}}(\phi) \leq C \varepsilon^{n-2} \rho_{0}^{2-n}
$$

Now let us turn our attention to the quantity $E_{B_{\rho_{0}}^{+}}(\phi)$. Since $N$ is as large as we want in equality (3.2), we can just assume $d v_{g}=d x d t$ in the expansions to come.

Using that $g_{t t}=1$ and $g_{t i}=0$ in Fermi coordinates, we obtain

$$
\int_{B_{\rho_{0}}^{+}}|\nabla \phi|_{g}^{2} d x d t=\int_{B_{\rho_{0}}^{+}}\left|\nabla u_{\varepsilon}\right|^{2} d x d t+\int_{B_{\rho_{0}}^{+}}\left(g^{i j}-\delta^{i j}\right)\left(u_{\varepsilon}\right)_{i}\left(u_{\varepsilon}\right)_{j} d x d t
$$

where the indices on functions denote derivatives. 
Now

$$
\int_{B_{\rho_{0}}^{+}}\left|\nabla u_{\varepsilon}\right|^{2} d x d t=\int_{B_{\rho_{0}}^{+}}\left(\left|\nabla U_{\varepsilon}\right|^{2}+\left|\nabla \psi_{\varepsilon}\right|^{2}\right) d x d t .
$$

We are using that

$$
\int_{B_{\rho_{0}}^{+}}\left\langle\nabla U_{\varepsilon}, \nabla \psi_{\varepsilon}\right\rangle d x d t=0
$$

since

$$
\int_{S_{r}^{n-2}} h_{i j} x_{i} x_{j}=\frac{H}{n-1} \int_{S_{r}^{n-2}} r^{2}=0
$$

Note that from the properties (2.2) and the identity (2.3), we can get

$$
\int_{B_{\rho_{0}}^{+}}\left|\nabla U_{\varepsilon}\right|^{2} d x d t \leq Q\left(B^{n}, \partial B^{n}\right)\left(\int_{B_{\rho_{0}}^{n-1}} U_{\varepsilon}^{2(n-1) / n-2} d x\right)^{n-2 / n-1}
$$

Here, we are using that $\frac{\partial U_{\varepsilon}}{\partial \eta}<0$ on $\partial B_{\rho_{0}}^{+} \cap \mathbb{R}_{+}^{n}$, where $\eta$ denotes the outward unit normal vector.

On the other hand

$$
u_{\varepsilon}(x, 0)=U_{\varepsilon}(x, 0)
$$

Hence

$$
\int_{B_{\rho_{0}}^{+}}\left|\nabla U_{\varepsilon}\right|^{2} d x d t \leq Q\left(B^{n}, \partial B^{n}\right)\left(\int_{M} \phi^{2(n-1) / n-2} d \sigma_{g}\right)^{n-2 / n-1}
$$

Now integration by parts gives

$$
\begin{aligned}
\int_{B_{\rho_{0}}^{+}}\left|\nabla \psi_{\varepsilon}\right|^{2} d x d t= & -\int_{B_{\rho_{0}}^{+}} \psi_{\varepsilon} \Delta \psi_{\varepsilon} d x d t \\
& +\int_{S_{\rho_{0}}^{+}} \psi_{\varepsilon} \frac{\partial \psi_{\varepsilon}}{\partial r} d \sigma_{\rho_{0}}-\int_{B_{\rho_{0}}^{n-1}} \psi_{\varepsilon} \frac{\partial \psi_{\varepsilon}}{\partial t} d x \\
= & -\int_{B_{\rho_{0}}^{+}} \psi_{\varepsilon} \Delta \psi_{\varepsilon} d x d t+O\left(\varepsilon^{n-2} \rho_{0}^{4-n}\right)
\end{aligned}
$$

We are using the definition of $\psi_{\varepsilon}$ to estimate the integral over the upper hemisphere $S_{\rho_{0}}^{+}$and to observe that the integral over $B_{\rho_{0}}^{n-1}$ vanishes.

Let us now compute $\Delta \psi_{\varepsilon}$. 
If $Z=(\varepsilon+t)^{2}+|x|^{2}$, then $\Delta Z^{-n / 2}=2 n Z^{-(n+2) / 2}$ and

$$
\begin{aligned}
\Delta \psi_{\varepsilon}= & A \varepsilon^{(n-2) / 2}\left\{\Delta\left(h_{j k} x_{j} x_{k} t\right) Z^{-n / 2}-n\left\langle\nabla\left(h_{j k} x_{j} x_{k} t\right), Z^{-(n / 2)-1} \nabla Z\right\rangle\right. \\
& \left.+h_{j k} x_{j} x_{k} t \Delta\left(Z^{-n / 2}\right)\right\} \\
= & -2 n A \varepsilon^{(n-2) / 2} h_{j k} x_{j} x_{k} Z^{-(n+2) / 2}(2 t+\varepsilon) .
\end{aligned}
$$

We will use that

$$
\int_{S_{r}^{n-2}} q=\frac{r^{2}}{d(d+n-3)} \int_{S_{r}^{n-2}} \Delta q
$$

if $q$ is a homogeneous polynomial of degree $d$.

Therefore,

$$
\int_{S_{r}^{n-2}} h_{i j} h_{k l} x_{i} x_{j} x_{k} x_{l}=\frac{2\left(h_{i j}\right)^{2}}{(n-1)(n+1)} \int_{S_{r}^{n-2}} r^{4}
$$

and then, from identity (3.4),

$$
\begin{aligned}
\int_{B_{\rho_{0}}^{+}}\left|\nabla \psi_{\varepsilon}\right|^{2} d x d t= & 2 n A^{2} \varepsilon^{n-2} \int_{B_{\rho_{0}}^{+}} \frac{h_{i j} h_{k l} x_{i} x_{j} x_{k} x_{l} t(2 t+\varepsilon)}{\left((\varepsilon+t)^{2}+|x|^{2}\right)^{n+1}} d x d t \\
& +O\left(\varepsilon^{n-2} \rho_{0}^{4-n}\right) \\
= & \frac{4 n A^{2} \varepsilon^{n-2} \sum_{i, j}\left(h_{i j}\right)^{2}}{(n-1)(n+1)} \int_{B_{\rho_{0}}^{+}} \frac{|x|^{4} t(2 t+\varepsilon)}{\left((\varepsilon+t)^{2}+|x|^{2}\right)^{n+1}} d x d t \\
& +O\left(\varepsilon^{n-2} \rho_{0}^{4-n}\right) .
\end{aligned}
$$

Now,

$$
\begin{aligned}
& \int_{B_{\rho_{0}}^{+}}\left(g^{i j}-\delta^{i j}\right)\left(u_{\varepsilon}\right)_{i}\left(u_{\varepsilon}\right)_{j} d x d t \\
& \quad=\int_{B_{\rho_{0}}^{+}}\left(g^{i j}-\delta^{i j}\right)\left\{\left(U_{\varepsilon}\right)_{i}\left(U_{\varepsilon}\right)_{j}+2\left(U_{\varepsilon}\right)_{i}\left(\psi_{\varepsilon}\right)_{j}\right\} d x d t+E_{1}
\end{aligned}
$$

where

$$
E_{1}=\left\{\begin{array}{llc}
O\left(\varepsilon^{2} \rho_{0}\right) & \text { if } & n=4 \\
O\left(\varepsilon^{3} \log \left(\frac{\rho_{0}}{\varepsilon}\right)\right) & \text { if } & n=5 \\
O\left(\varepsilon^{3}\right) & \text { if } & n \geq 6
\end{array}\right.
$$


To see this note that

$$
\begin{aligned}
E_{1} & =\int_{B_{\rho_{0}}^{+}}\left(g^{i j}-\delta^{i j}\right)\left(\psi_{\varepsilon}\right)_{i}\left(\psi_{\varepsilon}\right)_{j} d x d t \\
& =\varepsilon^{n-2} O\left(\int_{B_{\rho_{0}}^{+}} \frac{|(x, t)|^{5}}{\left((\varepsilon+t)^{2}+|x|^{2}\right)^{n}} d x d t\right)
\end{aligned}
$$

and perform the change of variables ([7], Lemma 3.4):

$$
\int_{B_{\rho_{0}}^{+}} \frac{t^{k} x^{\alpha} d x d t}{\left((\varepsilon+t)^{2}+|x|^{2}\right)^{n-l}}=\varepsilon^{k+m+2 l-n} \int_{B_{\frac{\rho_{0}}{\varepsilon}}^{+}} \frac{s^{k} y^{\alpha} d y d s}{\left((1+s)^{2}+|y|^{2}\right)^{n-l}}
$$

where $\alpha=\left(i_{1}, \ldots, i_{m}\right)$ and $x^{\alpha}=x_{i_{1}}, \ldots, x_{i_{m}}$.

From the expansion (3.1), we can compute

$$
g^{i j}(x, t)=\delta_{i j}+2 h_{i j} t
$$

$$
+\frac{1}{3} \bar{R}_{i k j l} x_{k} x_{l}+2 h_{i j, k} t x_{k}+\left(R_{t i t j}+3 h_{i k} h_{k j}\right) t^{2}+O\left(|(x, t)|^{3}\right) .
$$

We also have

$$
\left(U_{\varepsilon}\right)_{i}(x, t)=(2-n) \varepsilon^{(n-2) / 2} x_{i}\left(\frac{1}{(\varepsilon+t)^{2}+|x|^{2}}\right)^{n / 2} .
$$

Therefore, since $R_{t t}=-\sum_{i, j}\left(h_{i j}\right)^{2}$,

$$
\begin{aligned}
\int_{B_{\rho_{0}}^{+}} & \left(g^{i j}-\delta^{i j}\right)\left(U_{\varepsilon}\right)_{i}\left(U_{\varepsilon}\right)_{j} d x d t \\
= & \int_{B_{\rho_{0}}^{+}}\left(\left(R_{t i t j}+3 h_{i k} h_{k j}\right) t^{2}+O\left(|(x, t)|^{3}\right)\right)\left(U_{\varepsilon}\right)_{i}\left(U_{\varepsilon}\right)_{j} d x d t \\
= & (n-2)^{2} \varepsilon^{n-2} \int_{B_{\rho_{0}}^{+}} \frac{\left(R_{t i t j}+3 h_{i k} h_{k j}\right) t^{2} x_{i} x_{j}}{\left((\varepsilon+t)^{2}+|x|^{2}\right)^{n}} d x d t+E_{2} \\
= & \frac{(n-2)^{2}}{n-1} \varepsilon^{n-2}\left(R_{t t}+3 \sum_{i, j}\left(h_{i j}\right)^{2}\right) \int_{B_{\rho_{0}}^{+}} \frac{t^{2}|x|^{2}}{\left((\varepsilon+t)^{2}+|x|^{2}\right)^{n}} d x d t+E_{2} \\
= & \frac{2(n-2)^{2}}{n-1} \varepsilon^{n-2} \sum_{i, j}\left(h_{i j}\right)^{2} \int_{B_{\rho_{0}}^{+}} \frac{t^{2}|x|^{2}}{\left((\varepsilon+t)^{2}+|x|^{2}\right)^{n}} d x d t+E_{2},
\end{aligned}
$$


where

$$
E_{2}=\left\{\begin{array}{lll}
O\left(\varepsilon^{2} \rho_{0}\right) & \text { if } & n=4 \\
O\left(\varepsilon^{3} \log \left(\frac{\rho_{0}}{\varepsilon}\right)\right) & \text { if } & n=5 \\
O\left(\varepsilon^{3}\right) & \text { if } & n \geq 6
\end{array}\right.
$$

We are using symmetry arguments to observe that

$$
\int_{B_{\rho_{0}}^{+}}\left(2 h_{i j} t+\frac{1}{3} \bar{R}_{i k j l} x_{k} x_{l}+2 h_{i j, k} t x_{k}\right)\left(U_{\varepsilon}\right)_{i}\left(U_{\varepsilon}\right)_{j} d x d t=0 .
$$

On the other hand, by integrating by parts and observing that $\psi_{\varepsilon}=0$ when $t=0$ :

$$
\begin{aligned}
& 2 \int_{B_{\rho_{0}}^{+}}\left(g^{i j}-\delta^{i j}\right)\left(U_{\varepsilon}\right)_{i}\left(\psi_{\varepsilon}\right)_{j} d x d t \\
& \quad=2 \int_{B_{\rho_{0}}^{+}}\left(2 h_{i j} t+O\left(|(x, t)|^{2}\right)\right)\left(U_{\varepsilon}\right)_{i}\left(\psi_{\varepsilon}\right)_{j} d x d t \\
& \quad=-4 \int_{B_{\rho_{0}}^{+}} h_{i j} t\left(U_{\varepsilon}\right)_{i j} \psi_{\varepsilon} d x d t+4 \rho_{0}^{-1} \int_{S_{\rho_{0}}^{+}} h_{i j} t\left(U_{\varepsilon}\right)_{i} \psi_{\varepsilon} x_{j} d \sigma_{\rho_{0}}+E_{3},
\end{aligned}
$$

where

$$
E_{3}=\left\{\begin{array}{lll}
O\left(\varepsilon^{2} \rho_{0}\right) & \text { if } & n=4 \\
O\left(\varepsilon^{3} \log \left(\frac{\rho_{0}}{\varepsilon}\right)\right) & \text { if } & n=5 \\
O\left(\varepsilon^{3}\right) & \text { if } & n \geq 6
\end{array}\right.
$$

We can compute

$$
\begin{aligned}
\left(U_{\varepsilon}\right)_{i j}(x, t)= & (2-n) \varepsilon^{(n-2) / 2} \delta_{i j}\left((\varepsilon+t)^{2}+|x|^{2}\right)^{-n / 2} \\
& +n(n-2) \varepsilon^{(n-2) / 2} x_{i} x_{j}\left((\varepsilon+t)^{2}+|x|^{2}\right)^{-(n+2) / 2} .
\end{aligned}
$$

Therefore,

$$
\begin{aligned}
& 2 \int_{B_{\rho_{0}}^{+}}\left(g^{i j}-\delta^{i j}\right)\left(U_{\varepsilon}\right)_{i}\left(\psi_{\varepsilon}\right)_{j} d x d t \\
& \quad=-4 n(n-2) A \varepsilon^{n-2} \int_{B_{\rho_{0}}^{+}} \frac{h_{i j} h_{k l} x_{i} x_{j} x_{k} x_{l} t^{2}}{\left((\varepsilon+t)^{2}+|x|^{2}\right)^{n+1}} d x d t+E_{3}^{\prime} \\
& \quad=-\frac{8 n(n-2)}{(n-1)(n+1)} A \varepsilon^{n-2} \sum_{i, j}\left(h_{i j}\right)^{2} \int_{B_{\rho_{0}}^{+}} \frac{|x|^{4} t^{2}}{\left((\varepsilon+t)^{2}+|x|^{2}\right)^{n+1}} d x d t+E_{3}^{\prime},
\end{aligned}
$$

where $E_{3}^{\prime}=E_{3}+O\left(\varepsilon^{n-2} \rho_{0}^{4-n}\right)$. 
Now for the scalar curvature term

$$
\begin{aligned}
\frac{n-2}{4(n-1)} \int_{B_{\rho_{0}}^{+}} R_{g} u_{\varepsilon}^{2} d x d t & =\frac{n-2}{4(n-1)} \int_{B_{\rho_{0}}^{+}}\left(R_{g}(0)+O(|(x, t)|)\right) u_{\varepsilon}^{2} d x d t \\
& =-\frac{n-2}{4(n-1)} \sum_{i, j}\left(h_{i j}\right)^{2} \int_{B_{\rho_{0}}^{+}} u_{\varepsilon}^{2} d x d t+E_{4}
\end{aligned}
$$

where

$$
E_{4}=\left\{\begin{array}{lll}
O\left(\varepsilon^{2} \rho_{0}\right) & \text { if } & n=4 \\
O\left(\varepsilon^{3} \log \left(\frac{\rho_{0}}{\varepsilon}\right)\right) & \text { if } & n=5 \\
O\left(\varepsilon^{3}\right) & \text { if } & n \geq 6
\end{array}\right.
$$

We are using that $R_{g}(P)=-\sum_{i, j}\left(h_{i j}\right)^{2}$ in conformal Fermi coordinates.

Since $u_{\varepsilon}^{2}=U_{\varepsilon}^{2}+2 U_{\varepsilon} \psi_{\varepsilon}+\psi_{\varepsilon}^{2}$, we get

$$
\begin{gathered}
\frac{n-2}{4(n-1)} \int_{B_{\rho_{0}}^{+}} R_{g} u_{\varepsilon}^{2} d x d t=-\frac{n-2}{4(n-1)} \sum_{i, j}\left(h_{i j}\right)^{2} \int_{B_{\rho_{0}}^{+}} U_{\varepsilon}^{2} d x d t+E_{5} \\
=-\frac{n-2}{4(n-1)} \sum_{i, j}\left(h_{i j}\right)^{2} \varepsilon^{n-2} \int_{B_{\rho_{0}}^{+}} \frac{1}{\left((\varepsilon+t)^{2}+|x|^{2}\right)^{n-2}} d x d t+E_{5}
\end{gathered}
$$

where

$$
E_{5}=E_{4}-\frac{n-2}{4(n-1)} \sum_{i, j}\left(h_{i j}\right)^{2} \int_{B_{\rho_{0}}^{+}} \psi_{\varepsilon}^{2} d x d t
$$

After using the definition of $\psi_{\varepsilon}$ and performing the change of variables (3.5) on the integral above, we get

$$
E_{5}=\left\{\begin{array}{lll}
O\left(\varepsilon^{2} \rho_{0}\right) & \text { if } & n=4 \\
O\left(\varepsilon^{3} \log \left(\frac{\rho_{0}}{\varepsilon}\right)\right) & \text { if } & n=5 \\
O\left(\varepsilon^{3}\right) & \text { if } & n \geq 6
\end{array}\right.
$$

The integral involving the mean curvature can be estimated as follows

$$
\int_{B_{\rho_{0}}^{n-1}} h_{g} u_{\varepsilon}^{2} d x=\int_{B_{\rho_{0}}^{n-1}} O\left(|x|^{2}\right) u_{\varepsilon}^{2} d x= \begin{cases}O\left(\varepsilon^{2} \rho_{0}\right) & \text { if } n=4 \\ O\left(\varepsilon^{3} \log \left(\frac{\rho_{0}}{\varepsilon}\right)\right) & \text { if } n=5 \\ O\left(\varepsilon^{3}\right) & \text { if } n \geq 6\end{cases}
$$

We are using that in conformal Fermi coordinates $H=H_{, k}=0$ at $P$. 
Therefore,

$$
\begin{aligned}
E(\phi) & \leq Q\left(B^{n}, \partial B^{n}\right)\left(\int_{\partial M} \phi^{2(n-1) /(n-2)} d \sigma_{g}\right)^{n-2 / n-1} \\
& +\frac{4 n A^{2} \varepsilon^{2} \sum_{i, j}\left(h_{i j}\right)^{2}}{(n-1)(n+1)}\left\{2 \int_{B_{\rho_{0} / \varepsilon}^{+}} \frac{|y|^{4} s^{2}}{\left((1+s)^{2}+|y|^{2}\right)^{n+1}} d y d s\right. \\
& \left.+\int_{B_{\rho_{0} / \varepsilon}^{+}} \frac{|y|^{4} s}{\left((1+s)^{2}+|y|^{2}\right)^{n+1}} d y d s\right\} \\
& -\frac{8 n(n-2)}{(n-1)(n+1)} A \varepsilon^{2} \sum_{i, j}\left(h_{i j}\right)^{2} \int_{B_{\rho_{0} / \varepsilon}^{+}} \frac{|y|^{4} s^{2}}{\left((1+s)^{2}+|y|^{2}\right)^{n+1}} d y d s \\
+ & \frac{2(n-2)^{2}}{n-1} \varepsilon^{2} \sum_{i, j}\left(h_{i j}\right)^{2} \int_{B_{\rho_{0} / \varepsilon}^{+}} \frac{s^{2}|y|^{2}}{\left((1+s)^{2}+|y|^{2}\right)^{n}} d y d s \\
& -\frac{n-2}{4(n-1)} \varepsilon^{2} \sum_{i, j}\left(h_{i j}\right)^{2} \int_{B_{\rho_{0} / \varepsilon}^{+}} \frac{1}{\left((1+s)^{2}+|y|^{2}\right)^{n-2}} d y d s+E^{\prime}
\end{aligned}
$$

where

$$
E^{\prime}=\left\{\begin{array}{llc}
O\left(\varepsilon^{2} \rho_{0}^{-2}\right) & \text { if } & n=4 \\
O\left(\varepsilon^{3} \log \left(\frac{\rho_{0}}{\varepsilon}\right)\right) & \text { if } & n=5 \\
O\left(\varepsilon^{3}\right) & \text { if } & n \geq 6
\end{array}\right.
$$

Let us divide the rest of the proof in two cases.

Case $n=5$. We will apply the change of variables $y=(1+s) z$ in order to compare the different integrals in expansion (3.7).

First

$$
\begin{aligned}
I_{1} & =\int_{\mathbb{R}_{+}^{n}} \frac{|y|^{4} s^{2}}{\left((1+s)^{2}+|y|^{2}\right)^{n+1}} d y d s \\
& =\int_{0}^{\infty} \frac{s^{2}}{(1+s)^{n-1}} d s \int_{\mathbb{R}^{n-1}} \frac{|z|^{4}}{\left(1+|z|^{2}\right)^{n+1}} d z \\
& =\frac{2 \sigma_{n-2}}{(n-2)(n-3)(n-4)} \int_{0}^{\infty} \frac{r^{n+2}}{\left(1+r^{2}\right)^{n+1}} d r
\end{aligned}
$$

where $\sigma_{n-2}$ denotes the volume of the $(n-2)$-dimensional unit sphere. 
For the second integral,

$$
\begin{aligned}
I_{2} & =\int_{\mathbb{R}_{+}^{n}} \frac{|y|^{4} s}{\left((1+s)^{2}+|y|^{2}\right)^{n+1}} d y d s \\
& =\int_{0}^{\infty} \frac{s}{(1+s)^{n-1}} d s \int_{\mathbb{R}^{n-1}} \frac{|z|^{4}}{\left(1+|z|^{2}\right)^{n+1}} d z \\
& =\frac{\sigma_{n-2}}{(n-2)(n-3)} \int_{0}^{\infty} \frac{r^{n+2}}{\left(1+r^{2}\right)^{n+1}} d r .
\end{aligned}
$$

Now the fourth integral,

$$
\begin{aligned}
I_{4} & =\int_{\mathbb{R}_{+}^{n}} \frac{|y|^{2} s^{2}}{\left((1+s)^{2}+|y|^{2}\right)^{n}} d y d s \\
& =\int_{0}^{\infty} \frac{s^{2}}{(1+s)^{n-1}} d s \int_{\mathbb{R}^{n-1}} \frac{|z|^{2}}{\left(1+|z|^{2}\right)^{n}} d z \\
& =\frac{2 \sigma_{n-2}}{(n-2)(n-3)(n-4)} \int_{0}^{\infty} \frac{r^{n}}{\left(1+r^{2}\right)^{n}} d r .
\end{aligned}
$$

And the last integral,

$$
\begin{aligned}
I_{5} & =\int_{\mathbb{R}_{+}^{n}} \frac{1}{\left((1+s)^{2}+|y|^{2}\right)^{n-2}} d y d s \\
& =\int_{0}^{\infty} \frac{d s}{(1+s)^{n-3}} \int_{\mathbb{R}^{n-1}} \frac{d z}{\left(1+|z|^{2}\right)^{n-2}} \\
& =\frac{\sigma_{n-2}}{n-4} \int_{0}^{\infty} \frac{r^{n-2}}{\left(1+r^{2}\right)^{n-2}} d r .
\end{aligned}
$$

Thus,

$$
\begin{aligned}
& E(\phi) \leq Q\left(B^{n}, \partial B^{n}\right)\left(\int_{\partial M} \phi^{2(n-1) /(n-2)} d \sigma_{g}\right)^{n-2 / n-1} \\
& +A^{2} \varepsilon^{2} \sum_{i, j}\left(h_{i j}\right)^{2}\left\{\frac{8 n}{(n-1)(n+1)} I_{1}+\frac{4 n}{(n-1)(n+1)} I_{2}\right\} \\
& +A \varepsilon^{2} \sum_{i, j}\left(h_{i j}\right)^{2}\left\{-\frac{8 n(n-2)}{(n-1)(n+1)} I_{1}\right\} \\
& +\varepsilon^{2} \sum_{i, j}\left(h_{i j}\right)^{2}\left\{\frac{2(n-2)^{2}}{n-1} I_{4}-\frac{n-2}{4(n-1)} I_{5}\right\}+E
\end{aligned}
$$


where

$$
E=O\left(\varepsilon^{3} \log \left(\frac{\rho_{0}}{\varepsilon}\right)\right) .
$$

We are using that the differences between the integrals in expansion (3.7) and the corresponding integrals over the entire $\mathbb{R}_{+}^{n}$ do not change the order of the error terms.

Now integration by parts yields

$$
\int_{0}^{\infty} \frac{r^{n}}{\left(1+r^{2}\right)^{n}} d r=\frac{2 n}{n+1} \int_{0}^{\infty} \frac{r^{n+2}}{\left(1+r^{2}\right)^{n+1}} d r .
$$

For the same reason

$$
\int_{0}^{\infty} \frac{r^{n-2}}{\left(1+r^{2}\right)^{n-2}} d r=\frac{2(n-2)}{n-1} \int_{0}^{\infty} \frac{r^{n}}{\left(1+r^{2}\right)^{n-1}} d r,
$$

and also

$$
\int_{0}^{\infty} \frac{r^{n}}{\left(1+r^{2}\right)^{n-1}} d r=\frac{2(n-1)}{n+1} \int_{0}^{\infty} \frac{r^{n+2}}{\left(1+r^{2}\right)^{n}} d r
$$

so

$$
\int_{0}^{\infty} \frac{r^{n-2}}{\left(1+r^{2}\right)^{n-2}} d r=\frac{4(n-2)}{n+1} \int_{0}^{\infty} \frac{r^{n+2}}{\left(1+r^{2}\right)^{n}} d r .
$$

Now identity (3.9) and the equality

$$
\int_{0}^{\infty} \frac{r^{n}}{\left(1+r^{2}\right)^{n-1}} d r=\int_{0}^{\infty} \frac{r^{n}}{\left(1+r^{2}\right)^{n}} d r+\int_{0}^{\infty} \frac{r^{n+2}}{\left(1+r^{2}\right)^{n}} d r
$$

immediately imply

$$
\int_{0}^{\infty} \frac{r^{n+2}}{\left(1+r^{2}\right)^{n}} d r=\frac{n+1}{n-3} \int_{0}^{\infty} \frac{r^{n}}{\left(1+r^{2}\right)^{n}} d r .
$$

Therefore,

$$
\int_{0}^{\infty} \frac{r^{n-2}}{\left(1+r^{2}\right)^{n-2}} d r=\frac{8 n(n-2)}{(n-3)(n+1)} \int_{0}^{\infty} \frac{r^{n+2}}{\left(1+r^{2}\right)^{n+1}} d r .
$$

Hence,

$$
\begin{aligned}
& I_{2}=\frac{n-4}{2} I_{1}, \\
& I_{4}=\frac{2 n}{n+1} I_{1}
\end{aligned}
$$


and

$$
I_{5}=\frac{4 n(n-2)^{2}}{n+1} I_{1}
$$

Thus

$$
\begin{aligned}
& E(\phi) \leq Q\left(B^{n}, \partial B^{n}\right)\left(\int_{\partial M} \phi^{2(n-1) /(n-2)} d \sigma_{g}\right)^{n-2 / n-1} \\
& +A^{2} \varepsilon^{2} \sum_{i, j}\left(h_{i j}\right)^{2} I_{1}\left\{\frac{8 n}{(n-1)(n+1)}+\frac{2 n(n-4)}{(n-1)(n+1)}\right\} \\
& -A \varepsilon^{2} \sum_{i, j}\left(h_{i j}\right)^{2} I_{1}\left\{\frac{8 n(n-2)}{(n-1)(n+1)}\right\} \\
& +\varepsilon^{2} \sum_{i, j}\left(h_{i j}\right)^{2} I_{1}\left\{\frac{4 n(n-2)^{2}}{(n-1)(n+1)}-\frac{n(n-2)^{3}}{(n-1)(n+1)}\right\}+E .
\end{aligned}
$$

Since $n=5$, we get

$$
\begin{aligned}
& E(\phi) \leq Q\left(B^{n}, \partial B^{n}\right)\left(\int_{\partial M} \phi^{2(n-1) /(n-2)} d \sigma_{g}\right)^{n-2 / n-1} \\
& \quad+\frac{5 \varepsilon^{2} \sum_{i, j}\left(h_{i j}\right)^{2} I_{1}}{24}\left\{10 A^{2}-24 A+9\right\}+C \varepsilon^{3} \log \left(\frac{\rho_{0}}{\varepsilon}\right) .
\end{aligned}
$$

Choosing $A=1$, and observing that $\sum_{i, j}\left(h_{i j}\right)^{2}(P)>0$ since $P \in \partial M$ is not umbilic, we obtain

$$
E(\phi)<Q\left(B^{n}, \partial B^{n}\right)\left(\int_{\partial M} \phi^{2(n-1) /(n-2)} d \sigma_{g}\right)^{n-2 / n-1} \quad \text { for small } \varepsilon
$$

This finishes the proof when $n=5$.

Case $n=4$. We will again apply the change of variables $y=(1+s) z$ to the integrals in expansion (3.7).

We should point out that we will always assume $n=4$ in the following estimates. 
For the first integral

$$
\begin{aligned}
I_{1, \rho_{0} / \varepsilon} & =\int_{B_{\rho_{0} / \varepsilon}^{+}} \frac{|y|^{4} s^{2}}{\left((1+s)^{2}+|y|^{2}\right)^{n+1}} d y d s \\
& =\int_{B_{\rho_{0} / \varepsilon}^{+} \cap\left\{s \leq \rho_{0} / 2 \varepsilon\right\}} \frac{|y|^{4} s^{2}}{\left((1+s)^{2}+|y|^{2}\right)^{n+1}} d y d s+O(1) \\
& =\int_{\mathbb{R}_{+}^{n} \cap\left\{s \leq \rho_{0} / 2 \varepsilon\right\}} \frac{|y|^{4} s^{2}}{\left((1+s)^{2}+|y|^{2}\right)^{n+1}} d y d s+O(1) .
\end{aligned}
$$

Hence,

$$
\begin{aligned}
I_{1, \rho_{0} / \varepsilon} & =\int_{0}^{\rho_{0} / 2 \varepsilon} \frac{s^{2}}{(1+s)^{n-1}} d s \int_{\mathbb{R}^{n-1}} \frac{|z|^{4}}{\left(1+|z|^{2}\right)^{n+1}} d z+O(1) \\
& =\left(\sigma_{n-2} \int_{0}^{\infty} \frac{r^{n+2}}{\left(1+r^{2}\right)^{n+1}} d r\right) \log \left(\frac{\rho_{0}}{\varepsilon}\right)+O(1) .
\end{aligned}
$$

The second integral

$$
I_{2, \rho_{0} / \varepsilon}=\int_{B_{\rho_{0} / \varepsilon}^{+}} \frac{|y|^{4} s}{\left((1+s)^{2}+|y|^{2}\right)^{n+1}} d y d s=O(1)
$$

Now the fourth integral

$$
\begin{aligned}
I_{4, \rho_{0} / \varepsilon} & =\int_{B_{\rho_{0} / \varepsilon}^{+}} \frac{|y|^{2} s^{2}}{\left((1+s)^{2}+|y|^{2}\right)^{n}} d y d s \\
& =\int_{B_{\rho_{0} / \varepsilon}^{+} \cap\left\{s \leq \rho_{0} / 2 \varepsilon\right\}} \frac{|y|^{2} s^{2}}{\left((1+s)^{2}+|y|^{2}\right)^{n}} d y d s+O(1) \\
& =\int_{\mathbb{R}_{+}^{n} \cap\left\{s \leq \rho_{0} / 2 \varepsilon\right\}} \frac{|y|^{2} s^{2}}{\left((1+s)^{2}+|y|^{2}\right)^{n}} d y d s+O(1) \\
& =\int_{0}^{\rho_{0} / 2 \varepsilon} \frac{s^{2}}{(1+s)^{n-1}} d s \int_{\mathbb{R}^{n-1}} \frac{|z|^{2}}{\left(1+|z|^{2}\right)^{n}} d z+O(1) \\
& =\left(\sigma_{n-2} \int_{0}^{\infty} \frac{r^{n}}{\left(1+r^{2}\right)^{n}} d r\right) \log \left(\frac{\rho_{0}}{\varepsilon}\right)+O(1) .
\end{aligned}
$$


Similarly,

$$
\begin{aligned}
I_{5, \rho_{0} / \varepsilon} & =\int_{B_{\rho_{0} / \varepsilon}^{+}} \frac{1}{\left((1+s)^{2}+|y|^{2}\right)^{n-2}} d y d s \\
& =\int_{B_{\rho_{0} / \varepsilon}^{+} \cap\left\{s \leq \rho_{0} / 2 \varepsilon\right\}} \frac{1}{\left((1+s)^{2}+|y|^{2}\right)^{n-2}} d y d s+O(1) \\
& =\int_{\mathbb{R}_{+}^{n} \cap\left\{s \leq \rho_{0} / 2 \varepsilon\right\}} \frac{1}{\left((1+s)^{2}+|y|^{2}\right)^{n-2}} d y d s+O(1) \\
& =\int_{0}^{\rho_{0} / 2 \varepsilon} \frac{d s}{(1+s)^{n-3}} \int_{\mathbb{R}^{n-1}} \frac{d z}{\left(1+|z|^{2}\right)^{n-2}}+O(1) \\
& =\left(\sigma_{n-2} \int_{0}^{\infty} \frac{r^{n-2}}{\left(1+r^{2}\right)^{n-2}} d r\right) \log \left(\frac{\rho_{0}}{\varepsilon}\right)+O(1) .
\end{aligned}
$$

Thus,

$$
\begin{aligned}
E(\phi) & \leq Q\left(B^{n}, \partial B^{n}\right)\left(\int_{\partial M} \phi^{2(n-1) /(n-2)} d \sigma_{g}\right)^{n-2 / n-1} \\
& +A^{2} \varepsilon^{2} \sum_{i, j}\left(h_{i j}\right)^{2}\left\{\frac{8 n}{(n+1)(n-1)} I_{1, \rho_{0} / \varepsilon}\right\} \\
& -A \varepsilon^{2} \sum_{i, j}\left(h_{i j}\right)^{2}\left\{\frac{8 n(n-2)}{(n-1)(n+1)} I_{1, \rho_{0} / \varepsilon}\right\} \\
& +\varepsilon^{2} \sum_{i, j}\left(h_{i j}\right)^{2}\left\{\frac{2(n-2)^{2}}{n-1} I_{4, \rho_{0} / \varepsilon}-\frac{n-2}{4(n-1)} I_{5, \rho_{0} / \varepsilon}\right\}+O\left(\varepsilon^{2} \rho_{0}^{-2}\right) .
\end{aligned}
$$

Define

$$
J=\sigma_{n-2} \int_{0}^{\infty} \frac{r^{n+2}}{\left(1+r^{2}\right)^{n+1}} d r .
$$

Therefore, using identities (3.8) and (3.10):

$$
\begin{aligned}
& E(\phi) \leq Q\left(B^{n}, \partial B^{n}\right)\left(\int_{\partial M} \phi^{2(n-1) /(n-2)} d \sigma_{g}\right)^{n-2 / n-1} \\
& +\sum_{i, j}\left(h_{i j}\right)^{2} J \varepsilon^{2} \log \left(\frac{\rho_{0}}{\varepsilon}\right)\left\{\frac{8 n A^{2}}{(n+1)(n-1)}-\frac{8 n(n-2) A}{(n-1)(n+1)}\right. \\
& \left.\quad+\frac{4 n(n-2)^{2}}{(n-1)(n+1)}-\frac{8 n(n-2)^{2}}{4(n-3)(n-1)(n+1)}\right\}+O\left(\varepsilon^{2} \rho_{0}^{-2}\right) .
\end{aligned}
$$


Since $n=4$, we get

$$
\begin{aligned}
& E(\phi) \leq Q\left(B^{n}, \partial B^{n}\right)\left(\int_{\partial M} \phi^{2(n-1) /(n-2)} d \sigma_{g}\right)^{n-2 / n-1} \\
& +\frac{32}{15} \sum_{i, j}\left(h_{i j}\right)^{2} J \varepsilon^{2} \log \left(\frac{\rho_{0}}{\varepsilon}\right)\left\{A^{2}-2 A+1\right\}+O\left(\varepsilon^{2} \rho_{0}^{-2}\right) .
\end{aligned}
$$

The optimal choice is $A=1$, by which we lose the logarithmic singularity,

$$
E(\phi) \leq Q\left(B^{n}, \partial B^{n}\right)\left(\int_{\partial M} \phi^{2(n-1) /(n-2)} d \sigma_{g}\right)^{n-2 / n-1}+O\left(\varepsilon^{2} \rho_{0}^{-2}\right)
$$

In order to solve the problem, we will need to consider a small perturbation of $\psi_{\varepsilon}$ :

$$
\psi_{\varepsilon, \delta}=\psi_{\varepsilon}+\delta T
$$

where

$$
T=\varepsilon^{n-2 / 2} h_{i j} x_{i} x_{j}\left(\frac{1}{(\varepsilon+t)^{2}+|x|^{2}}\right)^{n-1 / 2} .
$$

Define $u_{\varepsilon}=U_{\varepsilon}+\psi_{\varepsilon, \delta}$ and $\phi=\eta u_{\varepsilon}$ as before.

Following the previous computations we get,

$$
\begin{aligned}
& E(\phi) \leq Q\left(B^{n}, \partial B^{n}\right)\left(\int_{\partial M} \phi^{2(n-1) /(n-2)} d \sigma_{g}\right)^{n-2 / n-1} \\
& +2 \delta \int_{B_{\rho_{0}}^{+}}\left\langle\nabla \psi_{\varepsilon}, \nabla T\right\rangle d x d t \\
& +4 \delta \int_{B_{\rho_{0}}^{+}} h_{i j} t\left(U_{\varepsilon}\right)_{i}(T)_{j} d x d t \\
& +\delta^{2} \int_{B_{\rho_{0}}^{+}}|\nabla T|^{2} d x d t+O\left(\varepsilon^{2} \rho_{0}^{-2}\right) .
\end{aligned}
$$

We are using that

$$
\int_{\partial M} \phi^{2(n-1) /(n-2)} d \sigma_{g} \geq \int_{B_{\rho_{0}}^{n-1}} u_{\varepsilon}^{2(n-1) /(n-2)} d \sigma_{g},
$$


and

$$
\begin{aligned}
\int_{B_{\rho_{0}}^{n-1}} & u_{\varepsilon}^{2(n-1) /(n-2)} d x \\
= & \int_{B_{\rho_{0}}^{n-1}}\left(U_{\varepsilon}+\delta T\right)^{2(n-1) /(n-2)} d x \\
= & \int_{B_{\rho_{0}}^{n-1}}\left(U_{\varepsilon}^{2(n-1) /(n-2)}+\frac{2(n-1)}{n-2} \delta T U_{\varepsilon}^{n / n-2}+\delta^{2} O\left(T^{2} U_{\varepsilon}^{2 / n-2}\right)\right) d x \\
= & \int_{B_{\rho_{0}}^{n-1}} U_{\varepsilon}^{2(n-1) /(n-2)} d x+O\left(\delta^{2} \varepsilon^{2}\right) .
\end{aligned}
$$

Now

$$
\begin{aligned}
2 \delta \int_{B_{\rho_{0}}^{+}}\left\langle\nabla \psi_{\varepsilon}, \nabla T\right\rangle d x d t+4 \delta \int_{B_{\rho_{0}}^{+}} h_{i j} t\left(U_{\varepsilon}\right)_{i}(T)_{j} d x d t \\
=-2 \delta \int_{B_{\rho_{0}}^{+}} T \Delta \psi_{\varepsilon} d x d t-4 \delta \int_{B_{\rho_{0}}^{+}} h_{i j} t\left(U_{\varepsilon}\right)_{i j} T d x d t \\
\quad+\delta \int_{S_{\rho_{0}}^{+}}\left\{2 T \frac{\partial \psi_{\varepsilon}}{\partial r}+4 \rho_{0}^{-1} h_{i j} t\left(U_{\varepsilon}\right)_{i} T x^{j}\right\} d \sigma_{\rho_{0}} \\
\quad-2 \delta \int_{B_{\rho_{0}}^{n-1}} T \frac{\partial \psi_{\varepsilon}}{\partial t} d x \\
=-2 \delta \int_{B_{\rho_{0}}^{+}} T\left(\Delta \psi_{\varepsilon}+2 h_{i j} t\left(U_{\varepsilon}\right)_{i j}\right) d x d t \\
\quad-2 A \delta \varepsilon^{n-2 / 2} \int_{B_{\rho_{0}}^{n-1}} T h_{j k} x_{j} x_{k} Z^{-n / 2} d x+\delta O\left(\varepsilon^{2}\right) .
\end{aligned}
$$

We are integrating by parts, estimating the integral over $S_{\rho_{0}}^{+}$, and using that

$$
\frac{\partial \psi_{\varepsilon}}{\partial t}=A \varepsilon^{n-2 / 2} h_{j k} x_{j} x_{k} Z^{-n / 2},
$$

when $t=0$. Recall $Z=(\varepsilon+t)^{2}+|x|^{2}$.

Note that

$$
\begin{aligned}
\Delta \psi_{\varepsilon} & +2 h_{i j} t\left(U_{\varepsilon}\right)_{i j}=-2 n A \varepsilon^{(n-2) / 2} h_{j k} x_{j} x_{k} Z^{-(n+2) / 2}(2 t+\varepsilon) \\
& +2 n(n-2) \varepsilon^{(n-2) / 2} h_{i j} x_{i} x_{j} t Z^{-(n+2) / 2} .
\end{aligned}
$$


Hence, if $n=4$ and $A=1$, after a cancellation we get,

$$
\Delta \psi_{\varepsilon}+2 h_{i j} t\left(U_{\varepsilon}\right)_{i j}=\varepsilon^{n / 2} O\left(|x|^{2} Z^{-(n+2) / 2}\right) .
$$

Therefore, in this case,

$$
\begin{aligned}
E(\phi) & \leq Q\left(B^{n}, \partial B^{n}\right)\left(\int_{\partial M} \phi^{2(n-1) /(n-2)} d \sigma_{g}\right)^{n-2 / n-1} \\
& -2 \delta \varepsilon^{n-2 / 2} \int_{B_{\rho_{0}}^{n-1}} T h_{j k} x_{j} x_{k} Z^{-n / 2} d x \\
& +\delta^{2} \int_{B_{\rho_{0}}^{+}}|\nabla T|^{2} d x d t+O\left(\varepsilon^{2} \rho_{0}^{-2}\right)
\end{aligned}
$$

From the definition (3.12),

$$
\begin{aligned}
E(\phi) & \leq Q\left(B^{n}, \partial B^{n}\right)\left(\int_{\partial M} \phi^{2(n-1) /(n-2)} d \sigma_{g}\right)^{n-2 / n-1} \\
& -\frac{4 \delta \varepsilon^{n-2}}{(n-1)(n+1)} \sum_{i, j}\left(h_{i j}\right)^{2} \int_{B_{\rho_{0}}^{n-1}} \frac{|x|^{4}}{\left(\varepsilon^{2}+|x|^{2}\right)^{n-1 / 2}} d x \\
& +\delta^{2} \int_{B_{\rho_{0}}^{+}}|\nabla T|^{2} d x d t+O\left(\varepsilon^{2} \rho_{0}^{-2}\right) .
\end{aligned}
$$

Hence,

$$
\begin{aligned}
E(\phi) & \leq Q\left(B^{n}, \partial B^{n}\right)\left(\int_{\partial M} \phi^{2(n-1) /(n-2)} d \sigma_{g}\right)^{n-2 / n-1} \\
& -\frac{4 \delta \varepsilon^{2}}{(n-1)(n+1)} \sum_{i, j}\left(h_{i j}\right)^{2} \int_{B_{\rho_{0} / \varepsilon}^{n-1}} \frac{|y|^{4}}{\left(1+|y|^{2}\right)^{n-1 / 2}} d y \\
& +O\left(\delta^{2} \varepsilon^{2} \log \left(\frac{\rho_{0}}{\varepsilon}\right)\right)+O\left(\varepsilon^{2} \rho_{0}^{-2}\right) .
\end{aligned}
$$

Therefore, for some $c>0$,

$$
\begin{aligned}
E(\phi) & \leq Q\left(B^{n}, \partial B^{n}\right)\left(\int_{\partial M} \phi^{2(n-1) /(n-2)} d \sigma_{g}\right)^{n-2 / n-1} \\
& -c \sum_{i, j}\left(h_{i j}\right)^{2} \delta \varepsilon^{2} \log \left(\frac{\rho_{0}}{\varepsilon}\right)+O\left(\delta^{2} \varepsilon^{2} \log \left(\frac{\rho_{0}}{\varepsilon}\right)\right)+O\left(\varepsilon^{2} \rho_{0}^{-2}\right) .
\end{aligned}
$$


Since $\sum_{i, j}\left(h_{i j}\right)^{2}>0$ at the nonumbilic point $P \in \partial M$, we find

$$
E(\phi)<Q\left(B^{n}, \partial B^{n}\right)\left(\int_{\partial M} \phi^{2(n-1) /(n-2)} d \sigma_{g}\right)^{n-2 / n-1}
$$

if $\delta>0$ is sufficiently small.

This finishes the proof of the Theorem.

\section{Acknowledgments}

Part of this work was written during the author's stay at Stanford University. The author would like to express his gratitude for the support received from CNPq-Brazil and Stanford University. He is also indebted to R. Schoen for the support and interest in the present work. Finally, he would like to thank the referees for the useful comments.

\section{References}

[1] M. Ould Ahmedou, A Riemann mapping type theorem in higher dimensions. I. The conformally flat case with umbilic boundary, in Nonlinear Equations: Methods, Models and Applications (Bergamo 2001), Progress Nonlinear Differential Equations Application, 54 Birkhäuser, 2003, 1-18.

[2] T. Aubin, Équations différentielles non linéaires et probléme de Yamabe concernant la courbure scalaire, J. Math. Pures Appl. 55 (1976), 269-296.

[3] W. Beckner, Sharp Sobolev inequalities on the sphere and the MoserTrudinger inequality, Ann. Math., (1993), 138(1):213-242.

[4] S. Brendle, A generalization of the Yamabe flow for manifolds with boundary, Asian J. Math. (2002), 6(4) 625-644.

[5] Z. Djadli, A. Malchiodi and M. Ould Ahmedou, Prescribing scalar and boundary mean curvature on the three-dimensional half sphere, J. Geom. Anal. 13(2) (2003), 255-289.

[6] J.F. Escobar, Sharp constant in a Sobolev trace inequality, Indiana Math. J. 37 (1988), 687-698. 
[7] J.F. Escobar, Conformal deformation of a Riemannian metric to a scalar flat metric with constant mean curvature on the boundary, Ann. Math. 136 (1992), 1-50.

[8] J.F. Escobar, The Yamabe problem on manifolds with boundary. J. Diff. Geom. 35 (1992), 21-84.

[9] J.F. Escobar, Addendum: conformal deformation of a Riemannian metric to a scalar flat metric with constant mean curvature on the boundary, Ann. Math. 139(3) (1994), 749-750.

[10] J.F. Escobar, Conformal deformation of a Riemannian metric to a constant scalar curvature metric with constant mean curvature on the boundary, Indiana Univ. Math. J. 45(4) (1996), 917-943.

[11] J.F. Escobar, Conformal metrics with prescribed mean curvature on the boundary, Calc. Var. 4 (1996), 559-592.

[12] J.F. Escobar and G. Garcia, Conformal metrics on the ball with zero scalar curvature and prescribed mean curvature on the boundary, J. Funct. Anal. 211(1) (2004), 71-152.

[13] V. Felli and M. Ould Ahmedou, Compactness results in conformal deformations of Riemannian metrics on manifolds with boundaries, Math. Z. 244(1) (2003), 175-210.

[14] V. Felli and M. Ould Ahmedou, Some geometric equations with critical nonlinearity at the boundary, Pac. J. Math. 218(1) (2005), 75-100.

[15] Z.C. Han and Y.Y. Li, The Yamabe problem on manifolds with boundary: existence and compactness results, Duke Math. J. 99(3) (1999), 489-542.

[16] Z.C. Han and Y.Y. Li, The existence of conformal metrics with constant scalar curvature and constant boundary mean curvature, Comm. Anal. Geom. 8(4) 2000, 809-869.

[17] E. Hebey and M. Vaugon, Le problème de Yamabe équivariant, Bull. Sci. Math. 117 (1993), 241-286.

[18] J. Lee and T. Parker, The Yamabe problem, Bull. Amer. Math. Soc. 17 (1987), 37-91.

[19] F.C. Marques, Existence results for the yamabe problem on manifolds with boundary, Indiana Univ. Math. J. 54(6) (2005), 1599-1620. 
[20] R. Schoen, Conformal deformation of a Riemannian metric to constant scalar curvature, J. Diff. Geom. 20 (1984), 479-495.

[21] N. Trudinger, Remarks concerning the conformal deformation of a Riemannian structure on compact manifolds, Ann. Scuola Norm. Sup. Pisa Cl. Sci. 22(3) (1968), 165-274.

[22] H. Yamabe, On a deformation of Riemannian structures on compact manifolds, Osaka Math. J. 12 (1960), 21-37.

Instituto de Matemática Pura e Aplicada (IMPA)

Estrada Dona Castorina 110 22460-320

RIO DE JANEIRO-RJ

BRAZIL

E-mail address: coda@impa.br

ReCeived March 29, 2006 
